\title{
ZUR PANEGYRISCHEN REDE DES THEOPHYLAKTOS VON ACHRIDA AUF KAISER ALEXIOS I. KOMNENOS*
}

Die panegyrische Rede des Theophylaktos von Achrida auf Kaiser Alexios I. Komnenos wurde von P. Gautier zuerst in RÉB 20 (1962) 93-130 (mit französischer Übersetzung) kritisch ediert. Eine Neuausgabe wurde von ihm dann 1980 im Rahmen des Corpus Fontium Historiae Byzantinae vorgelegt ${ }^{1}$. In einer ausführlichen Einleitung (S. 68-96) datiert Gautier diese Rede, aufgrund der historischen Angaben des Rhetors bezüglich der Innen- und Außenpolitik, überzeugend in das Jahr 1088 (den 6. Januar). Er widmet ein Kapitel jedem der vier wichtigsten Abschnitte der Rede: 1. westliche Politik, 2. orientalische Politik, 3. Innenpolitik und 4. Anna Dalassene und ihre politische Rolle. Es folgt die kritische Edition der Rede, die auf zwei Codices (L und F, beide Laurentiani) basiert. Die Ausgabe wird außer dem kritischen und dem Quellenapparat auch von einer französischen Übersetzung und Anmerkungen begleitet. ${ }^{2}$

Im folgenden werde ich einige Stellen der Rede besprechen, die auch in der neuen Ausgabe problematisch geblieben sind, und versuchen, diese zu korrigieren. Außerdem möchte ich auf einige zum Teil sehr wichtige Quellen des Autors aufmerksam machen, die Gautier und der späteren Forschung entgangen zu sein scheinen.

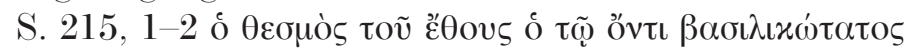

Es handelt sich um das kaiserliche Gesetz, dem gemäß jedes Jahr am Tag des Epiphanienfestes (am 6. Januar) ein berühmter Rhetor vor dem

* Für seine wertvollen Hinweise sei hier A. Kambylis noch einmal gedankt.

1 Théophylacte d'Achrida. Discours, traités, poésies. Introduction, texte, traduction et notes par P. Gautier (CFHB 16/1 - Series Thessalonicensis). Thessaloniki 1980. Die neue Ausgabe ist nicht in allen Punkten besser als die ältere, wie aus den folgenden Ausführungen ersichtlich wird. Hier gehe ich von der neuen Ausgabe aus. Von der älteren erwähne ich nur die Stellen, die in der neuen zu Unrecht geändert wurden.

${ }^{2}$ Die erste Ausgabe des Textes war diejenige von B. FinetTi, Theophylacti opera III. Venise 1758, die bei Migne nachgedruckt wurde (PG 126, 287-306); sie basiert auf einer Handschrift (Monac. gr. 66), die Gautier in seiner ersten Ausgabe neben L und F benutzt, in der Neuausgabe (S. 43) aber für ein Apograph des L hält. 
Kaiser und dem kaiserlichen Hof eine panegyrische Rede mit den Großtaten des Kaisers im vergangenen Jahr als Hauptthema hält. Gautier hat hier die Lesart éقous der Hs. L der Lesart étous der Hs. F vorgezogen, „parce que L transmet généralement un texte plus correct et surtout parce que la leçon retenue offre un sens plus obvie" . Der erstgenannte Grund ist schwach; dem zweiten stimme ich nicht zu, weil man hier eher den Sinn „das Gesetz des Jahres“, nämlich die jährlich stattfindende Veranstaltung bzw. die wichtigste Veranstaltung des Jahres, erwarten würde und nicht „das Gesetz der Gewohnheit"; letzterer Ausdruck scheint mir herabsetzend und für die Situation unpassend. Ich würde also die Lesart étovs in den

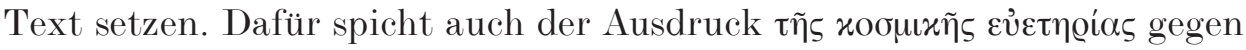
Ende der Rede (S. 241, 19-20). ${ }^{4}$

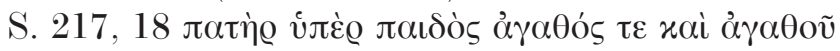

Die Lesart des Cod. F ảy $\alpha \theta$ oṽ $\tau \varepsilon$ xai $\alpha \gamma \alpha \theta$ òs scheint mir auch hier besser als die des Cod. L, da sie den rhetorischen Figuren des Chiasmus ${ }^{5}$ sowie des

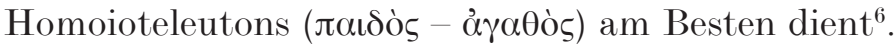

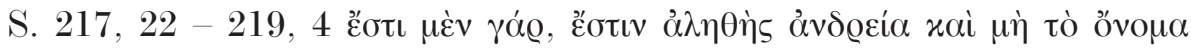

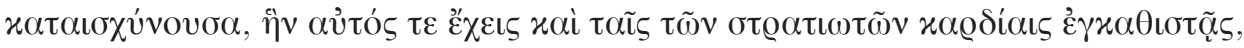

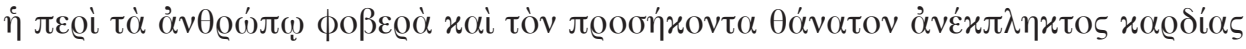

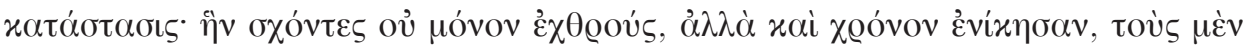

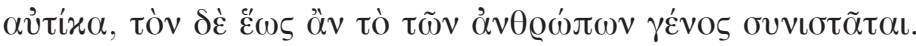

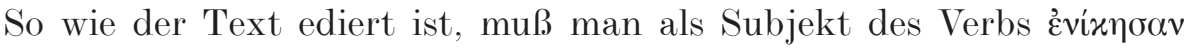

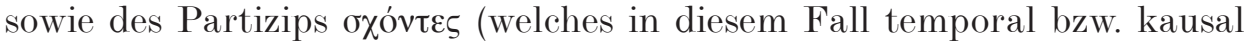

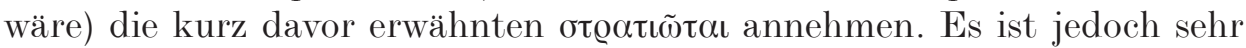
unwahrscheinlich, daß der Rhetor in einer Rede, die vor dem Kaiser gehalten wird, und zwar in einem Abschnitt, in dem die Rede von der Tapferkeit des Kaisers selbst ist (s. besonders Z. 19), plötzlich die tapferen Taten der Soldaten des Kaisers rühmt. Man erwartet vielmehr eine allgemeine Aussage über die Qualitäten der wahren Tapferkeit (Z. 22f.), welche der Kaiser besitzt und den Soldaten einzuflößen weiß. Die Schwierigkeit kann sehr

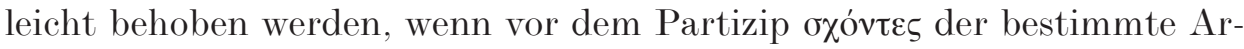

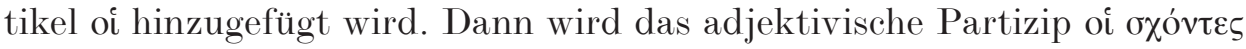

\footnotetext{
${ }^{3}$ S. 214, A. 1.

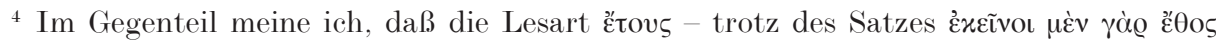

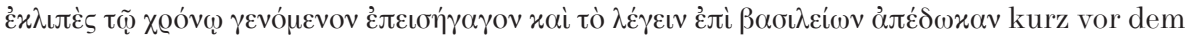
Ende der Rede (S. 243, 7-8) - aus den oben genannten Gründen vorzuziehen ist.

${ }^{5}$ Über die Vorliebe des Rhetors für solche Figuren vgl. in der selben Rede unten S. 221, $11-13$.

${ }^{6}$ In der älteren Ausgabe der Rede (s. o.) hat Gautier die Lesart des Cod. F in den Text gesetzt.
} 


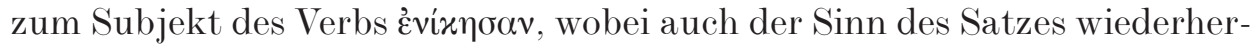
gestellt wird. Paläographisch kann die Auslassung des Artikels oi vor dem

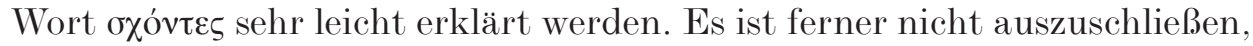
daß der Artikel überliefert ist, Gautier jedoch diesen mit dem $\sigma$ des darauf folgenden Partizips (das wahrscheinlich mit dem $\chi$ in einer Ligatur involviert ist) verwechselt hat. Wie dem auch sein mag, der Artikel oi gehört in den Text. Das Komma vor $\grave{\alpha} \lambda \lambda \dot{\alpha}$ kann ruhig weggelassen werden.

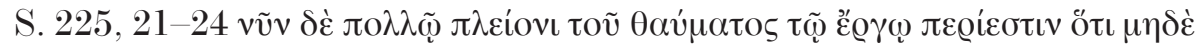

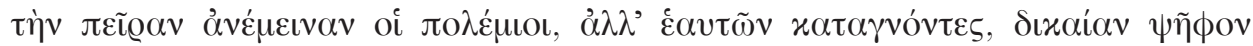

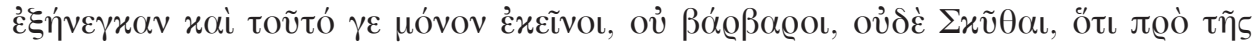

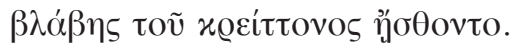

Problematisch ist hier die syntaktische Rolle des Dativs $\pi \lambda \varepsilon$ íovı. Ferner

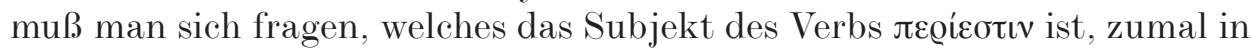
diesem Satz kein Nominativ vorhanden ist. Dieses Subjekt wird offensichtlich durch die auf das Verb folgenden ötı-Sätze erläutert. Der gesuchte Nominativ muß in dem merkwürdigen Dativ $\pi \lambda \varepsilon$ ciovı stecken. Das Problem wird durch die minimale Korrektur des korrupten $\pi \lambda \varepsilon_{\text {ciovı }}$ (es steht im Cod. $\mathrm{F}$; der Cod. L überliefert die ebenfalls korrupte Lesart $\pi \lambda \varepsilon_{\text {ciovo }}$ ) in den Nominativ des Neutrums $\pi \lambda \varepsilon \varepsilon_{0}$ v beseitigt. Paläographisch können beide Korruptelen sehr leicht erklärt werden: der Dativ ist durch Einfluß des

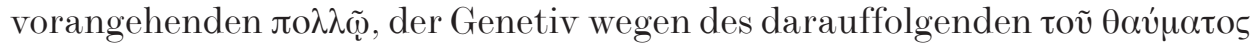
entstanden. Die Syntax und damit auch der Sinn des Hauptsatzes võv ḋ்

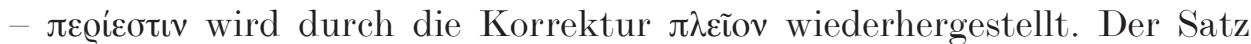
bedeutet etwa ,,jetzt aber gebührt dieser Tat viel mehr als Bewunderung“.

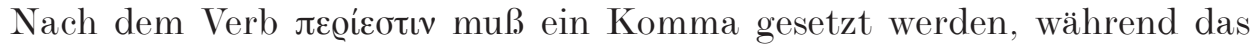

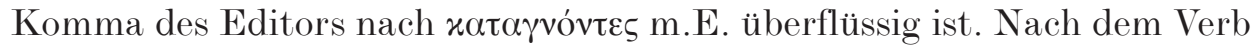
$\grave{\varepsilon} \xi \eta \dot{\eta} v \varepsilon$ r anfängt, der folgendermaßen übersetzt werden kann: „und nur insofern (waren) jene weder Barbaren noch Skythen, als sie das Bessere vor dem

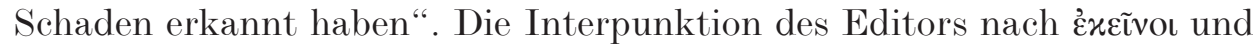
nach $\beta \alpha \dot{\varrho} \beta \alpha \varrho o r ~ m u ß ~ g e t i l g t$ werden. Der wiederhergestellte Text lautet: vũv

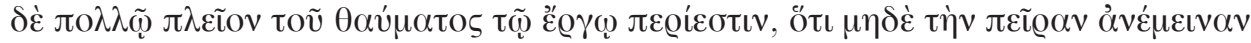

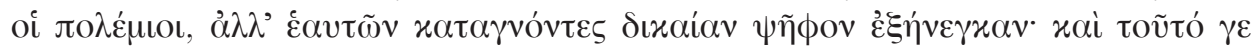

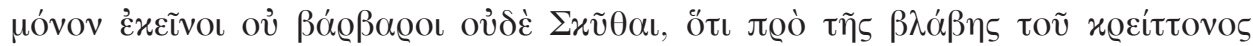

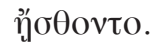

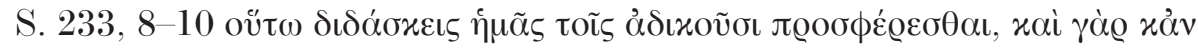

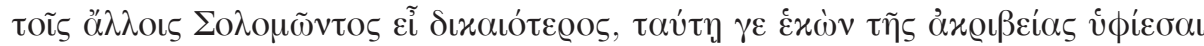

Obwohl Gautier hier richtig übersetzt, weist der edierte Text Probleme

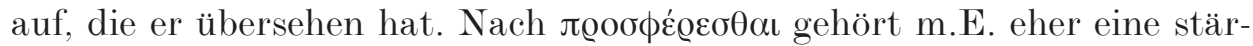
kere Interpunktion, z.B. ein Hochpunkt. Statt xỏv (= xai ह̉v) erwartet man hier die adversative Konjunktion xäv (= xai òv), die hier einen Konzessiv- 
satz einführt, wie auch Gautier richtig vermerkt hat: „même si à tous égards en effet tu es plus juste que Salomon"7. Über die bei den Byzantinern häufig vorkommende Konstruktion der Konjunktion xő้v mit Indikativ Präsens innerhalb eines Konzessivsatzes vgl. Fatouros ${ }^{8}$.

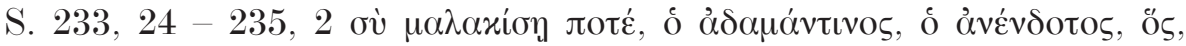

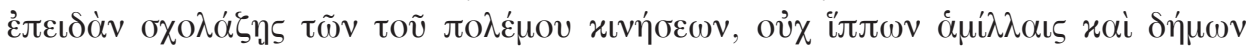

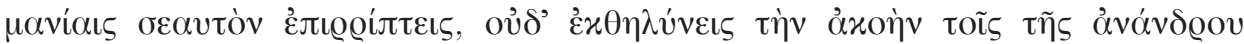

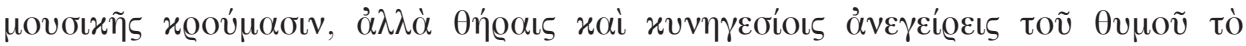

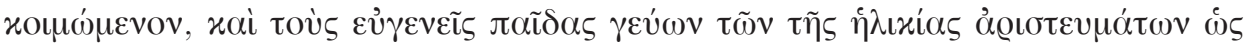

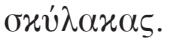

Diese Periode gibt nur dann einen guten Sinn, wenn sie als rhetorische Frage aufgefaßt wird. An ihr Ende gehört also ein Fragezeichen. Überflüssig und vielleicht auch irreführend ist ferner das Komma hinter dem Par-

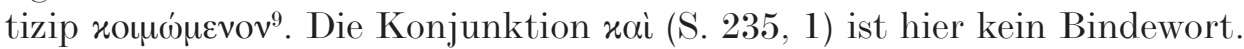
Sie bezieht sich nur auf das Objekt des Partizips $\gamma \varepsilon v ́ \omega v$ und bedeutet

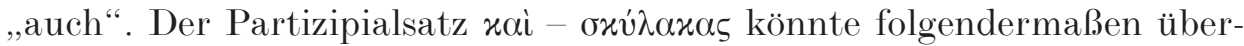
setzt werden: „indem Du auch die Jungen der edlen Familien die ihrem Alter passenden Errungenschaften schmecken läßt, als ob sie kleine Hunde wären".

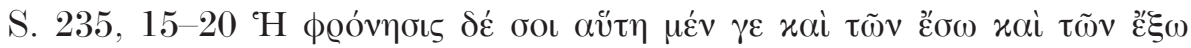

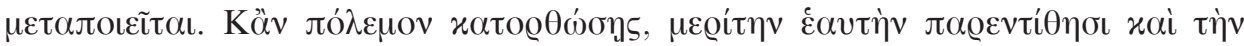

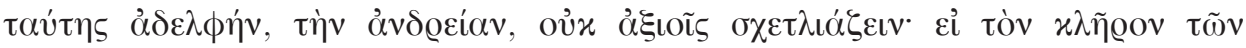

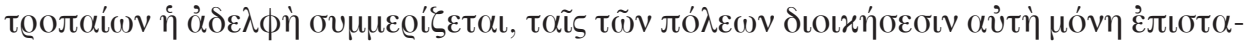

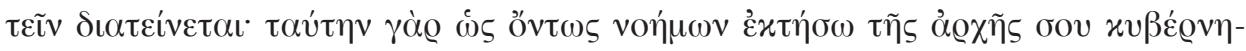
oiv.

In diesem Abschnitt ist die Rede von der allmächtigen ф@óvๆoıs, die alle Errungenschaften des Kaisers sowohl in der Innen- als auch in der Außenpolitik für sich beansprucht. Auch im Fall eines Sieges (bei dem normaler-

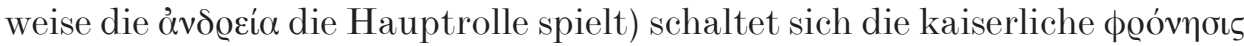
als berechtigt ein. Die Fortsetzung der Passage, wie sie ediert ist, scheint aber problematisch zu sein: Was besonders stört, ist die zweite Person $\dot{\alpha} \xi$ เoĩ

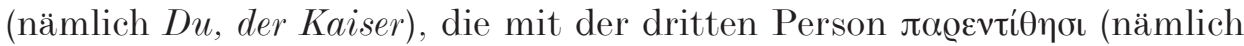

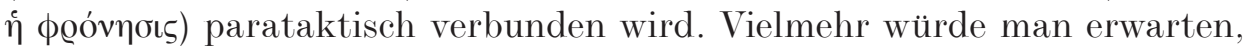
daß beide Verben dasselbe Subjekt hätten. In der Tat überliefert der Cod.

${ }^{7}$ In der früheren Ausgabe steht richtig xöv, was dafür spricht, daß xỏv hier ein Druck-

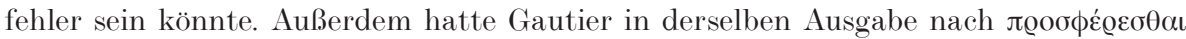
einen Punkt gesetzt.

8 Theodori Studitae Epistulae rec. G. Fatouros (CFHB 31/2). Berlin 1992, 898 Mitte (Index graecitatis).

${ }^{9}$ In der älteren Ausgabe fehlt das Komma. 
L die gewünschte Form der dritten Person $\grave{\alpha}_{\xi}$ เõ̃, die hier vorzuziehen ist ${ }^{10}$.

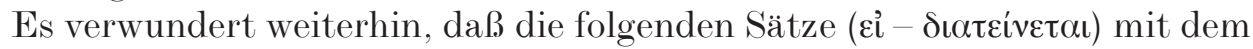
vorangehenden Text überhaupt nicht verbunden sind. Diese Schwierigkeit

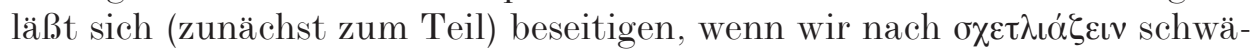

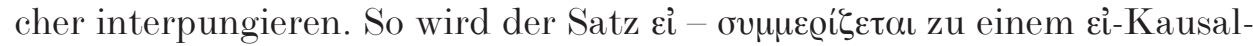

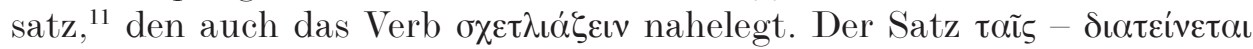
wiederum erscheint zwar als Hauptsatz, wird jedoch mit den vorangehenden Hauptsätzen auch nicht verbunden. Cod. F bietet aber hier, nach dem Artikel $\tau \alpha \tilde{s}$, die Konjunktion $\tau \varepsilon$. Dadurch wird der Satz mit dem vorigen

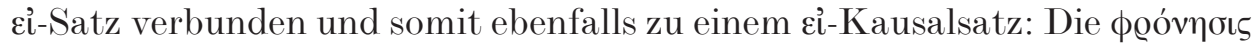
erlaubt ihrer Schwester $\alpha$ $v \delta \varrho \varepsilon i ́ \alpha$ nicht, sich darüber zu beschweren ${ }^{12}$, daß

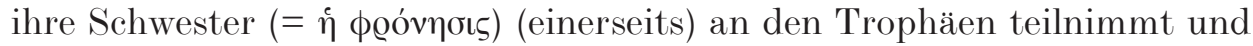
(andererseits) behauptet, daß sie allein der Verwaltung der Städte vorstehe. Leichte Interpunktion nach $\pi \alpha \varrho \varepsilon v \tau i \theta \eta \sigma \iota$ würde den Zusammenhang der übrigen Passage klarer machen. Auch am Anfang des Abschnitts ist Inter-

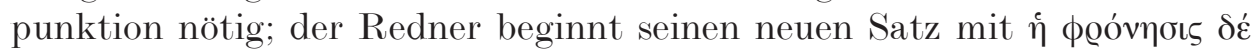

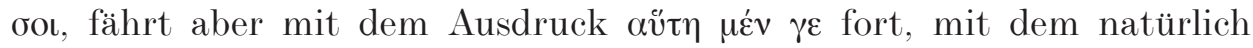

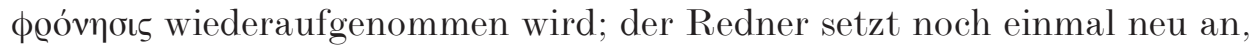
offenbar nach kurzer Pause (im Denken und Reden), die, wie ich meine, in der Edition durch ein Interpunktionszeichen kenntlich gemacht werden sollte. Ich habe mich für den Strich davor entschieden.

Im übrigen ist hier folgendes zu bemerken:

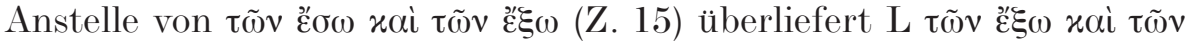
हैow. Angesichts der folgenden Zeilen, in denen die Rede zunächst vom Krieg (also eine Sache der Außenpolitik) und danach von der Regierung (also von Innenpolitik) ist, scheint die Reihenfolge $\left({ }^{\prime} \xi \omega-\varepsilon^{\prime} \sigma \omega\right)$ in L natürlicher ${ }^{13}$.

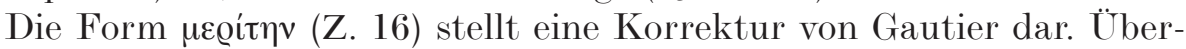
liefert ist (in beiden Codices) $\mu \varepsilon$ cítıv. Die feminine Endung kann, ja muß m.E. beibehalten werden ${ }^{14}$. Sonst gehen wir das Risiko ein, aufgrund unseres bisherigen Wissens und des Standes unserer Lexika den Autor selbst zu „,korrigieren"15. Allein über die Richtigkeit der Akzentuierung -ítıv dürfte

10 Das hatte Gautier in seiner ersten Ausgabe getan.

11 Zur Verwendung von ei anstelle des kausalen őtı s. LS.J s.v. عi B. V.

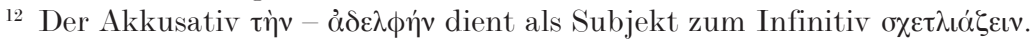

13 In seiner ersten Ausgabe hatte Gautier die Lesart des Cod. L in den Text gesetzt.

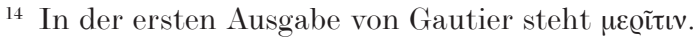

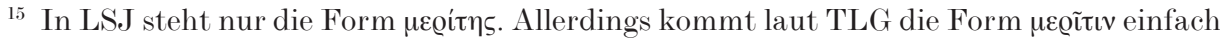
und als Kompositum mindestens noch je einmal vor: Const. Manass., Brev. Hist. Metr.

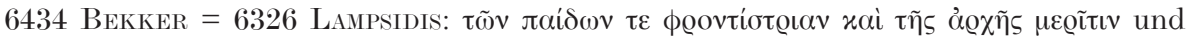

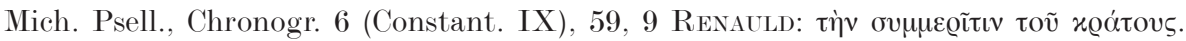
(Leider stand mir die neue Edition von Impellizzeri nicht zur Verfügung.) 
man zweifeln; da sich aber solche Erscheinungen in den byzantinischen Texten häufen ${ }^{16}$, sollte man nicht allzu schnell in das Überlieferte eingreifen.

Der wiederhergestellte Text lautet also:

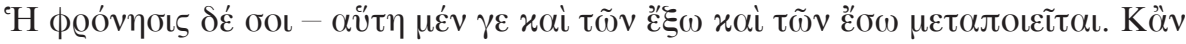

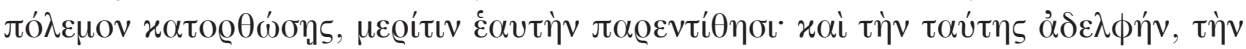

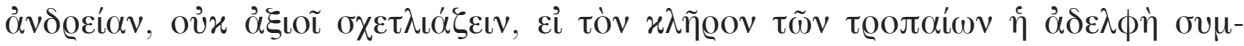

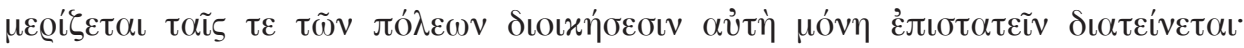

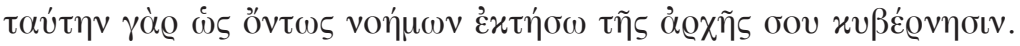

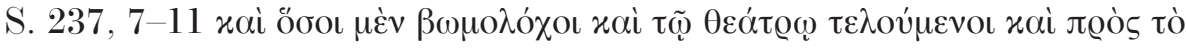

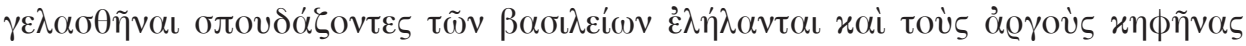

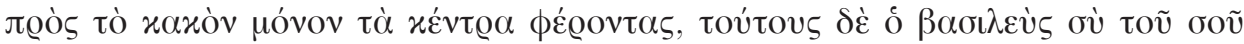

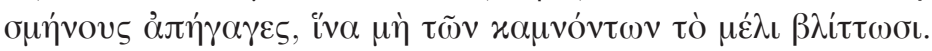

Nach $\dot{\varepsilon} \lambda \dot{\eta} \lambda \alpha v \tau \alpha$ gehört eine Interpunktion, entweder Komma oder Hochpunkt. Denn darauf folgt ein anderer Hauptsatz mit anderem Subjekt, der den Sinn des vorangehenden Satzes ergänzt. Der Relativsatz xai

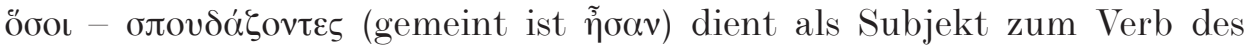
Hauptsatzes $\tau \tilde{\omega} v \beta \alpha \sigma i \lambda \varepsilon i \omega v$ ह่ $\lambda \dot{\eta} \lambda \alpha v \tau \alpha$.

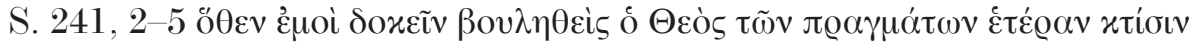

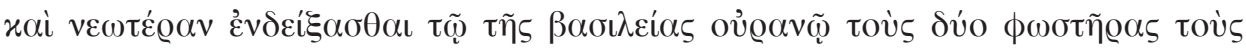

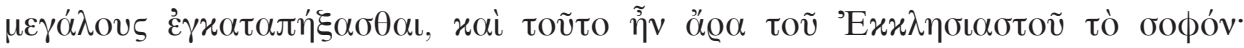

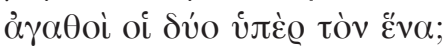

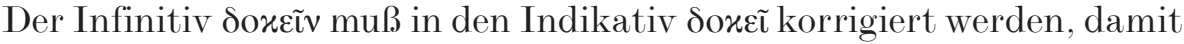
der Satz ein verbum finitum bekommt. Das Fragezeichen hinter dem Zitat muß getilgt werden; die Partikel ő@ $\alpha$ ist konsekutiv, führt also hier eine

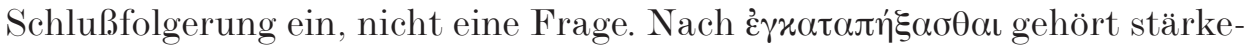
re Interpunktion ${ }^{17}$.

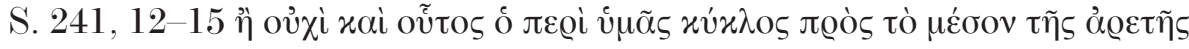

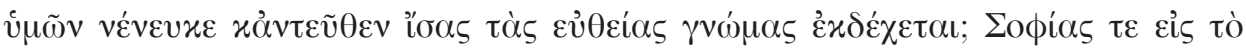

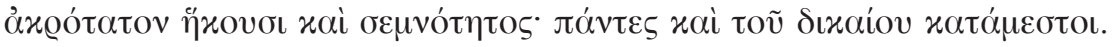

Hier wäre die Syntax „einfacher“ und der Sinn deutlicher, wenn wir anders interpungierten; vor ooфías $\tau \varepsilon$ braucht man eher ein Komma, da die

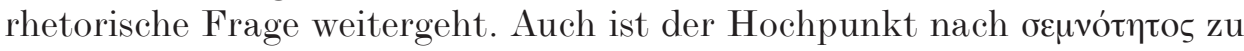

${ }^{16}$ In der handschriftlichen Überlieferung der Alexias von Anna Komnene erscheinen z.B.

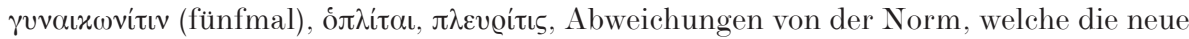
Ausgabe respektiert: Annae Comnenae, Alexias. Recensuerunt D. R. Reinsch et A. Kambylis. Pars prior, Prolegomena et textus. Pars altera, Indices, digesserunt F. KoLovou et D. R. Reinsch (CFHB 40, 1-2 - Series Berolinensis). Berlin-New York 2001. S. Index Graecitatis s.v. accentus.

${ }^{17}$ In der früheren Ausgabe sind alle diese Fehler nicht vorhanden. 


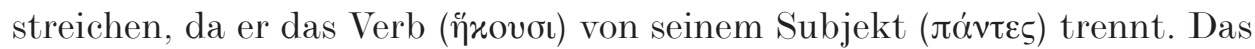

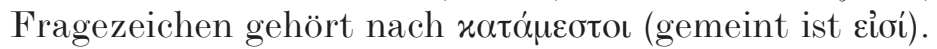

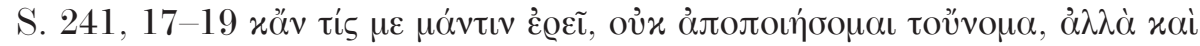

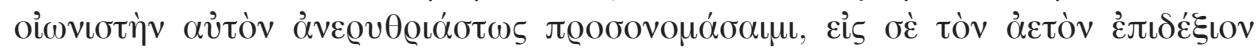
$\beta \lambda \dot{\varepsilon} \pi 0 v \tau \alpha$.

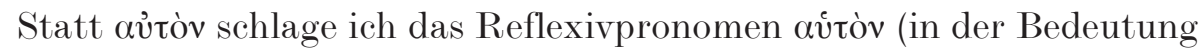

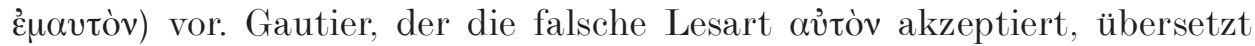
etwas ungenau: ,Que dis-je, j'accepterais même sans rougir le nom d'augure: $i l$ fixerait son regard sur toi, l'aigle d'heureux augure "18. Wenn man die Lesart aúcòv in den Text setzt, kann man folgendermaßen übersetzen: „,.. sondern ich würde mich selbst, ohne zu erröten, sogar Auguren nennen, der ich Dich beobachte, den Adler des guten Omens ${ }^{19 ،}$.

Im folgenden noch einige Stellen der Rede, die Gautier nicht richtig bzw. nicht genau übersetzt hat ${ }^{20}$ :

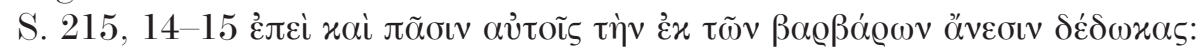
,ppuisque c'est à eux tous que tu as procuré la tranquillité en triomphant des barbares".

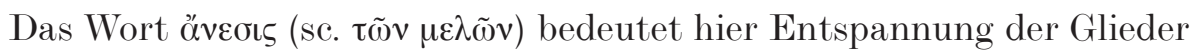
des Körpers, die vorher wegen der Kriegsgefahr schlaflos und gespannt waren.

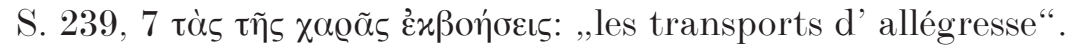

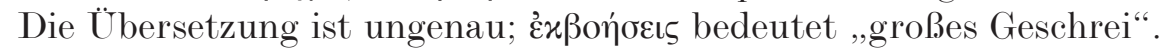

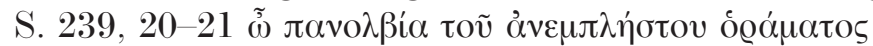

Gautier übersetzt „Ô femme réjouie par ce spectacle merveilleux“. Das Adjektiv $\pi \alpha v o \lambda \beta i ́ \alpha$ heißt aber ,ganz glücklich“, nicht bloß „,fröhlich“. Das

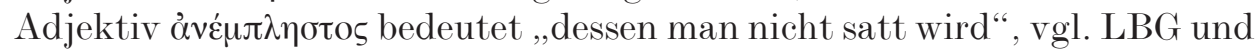
LS.J $^{21}$ s. $v$.

Ferner ist es vielleicht von gewissem Interesse, hier einige Quellen, die Gautier entgangen sind bzw. die er nicht identifiziert hat, sowie Paralleltexte, die zum sprachlichen Verständnis des Textes beitragen, zusammenzustellen:

18 In der früheren Ausgabe war die Übersetzung besser.

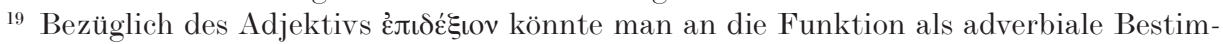
mung zu $\beta \lambda \varepsilon \dot{\tau} \tau o v \tau \alpha$ denken. Allerdings meine ich, daß in diesem Kontext ( $\mu \alpha \dot{v} \tau \iota v$,

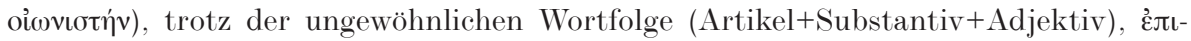

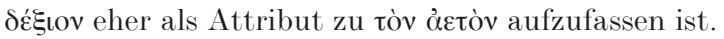

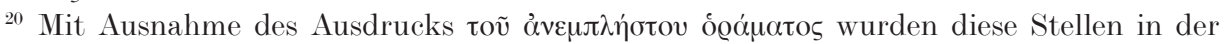
früheren Ausgabe besser übersetzt.

21 Laut LSJ ist das Wort allerdings nur als varia lectio bei Themistios belegt. Über die enge Beziehung des Theophylaktos-Textes zu Themistios siehe unten. 


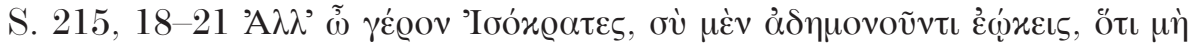

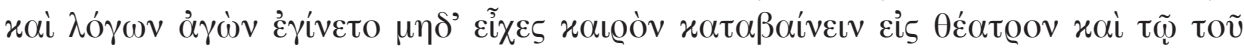

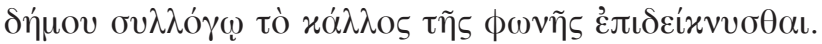

Der Rhetor bezieht sich wahrscheinlich auf den Panegyrikos des Iso-

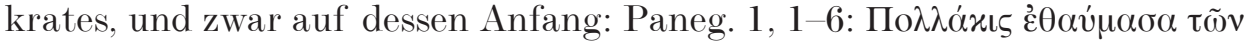

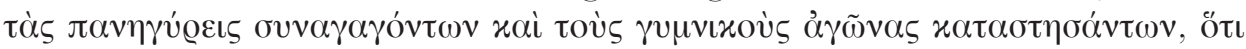

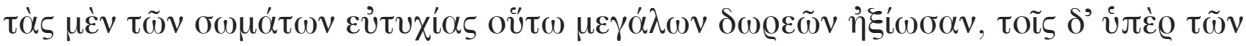

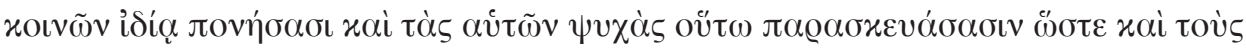

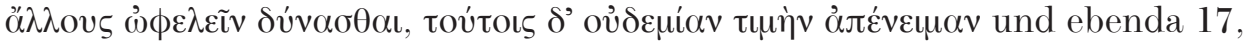

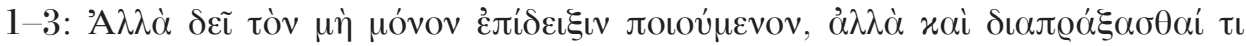

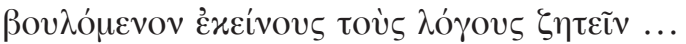

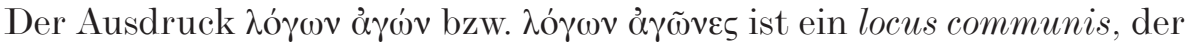
in TLG ca. 15mal belegt ist.

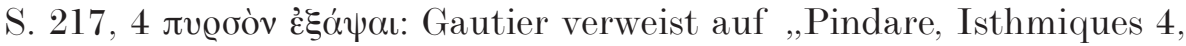
74“. Der Verweis muß in Pind. Isthm. III/IV 61 korrigiert werden.

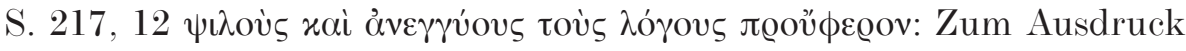

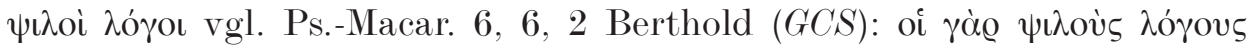

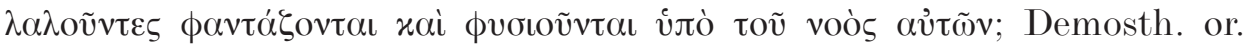

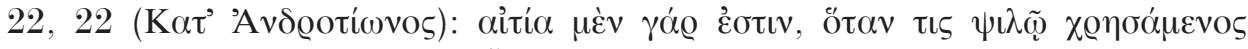

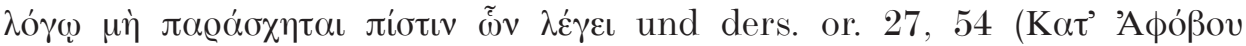

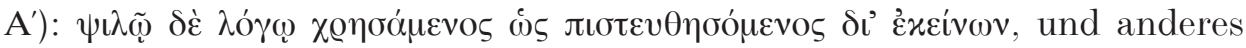
mehr.

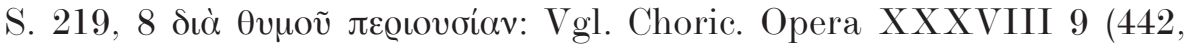

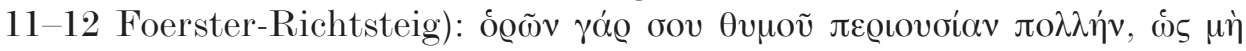

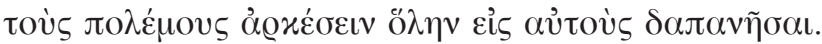

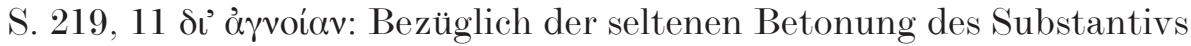
vgl. Catenam in S. Pauli ep. ad Rom. p. 177, 28 (Catenae Graec. patrum in

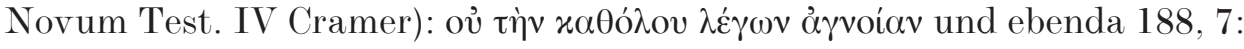

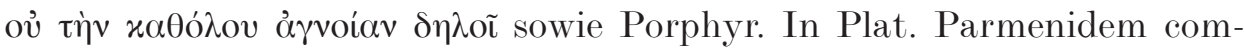
ment. V, 25-26 (Porphyre et Victorinus II 2 Hadot): [xai $\tau \grave{\eta}] v ~ \dot{\alpha}[\gamma v o i] \alpha v$

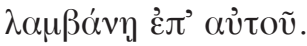

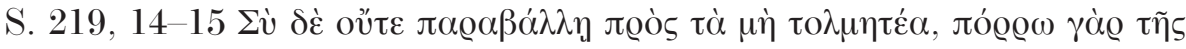

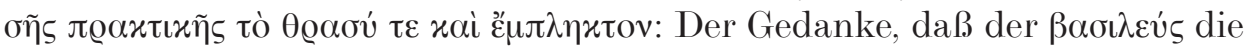
unbesonnene Kühnheit vermeiden muß, kommt, entweder als Rüge oder als Ratschlag, auch bei Isocrates Epist. 2 (an Philippos) vor, ep. 2. 3: Ov̉deis

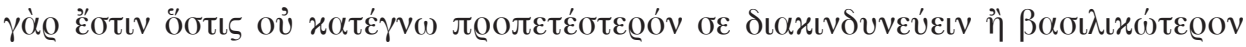

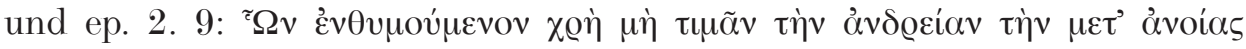

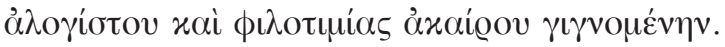

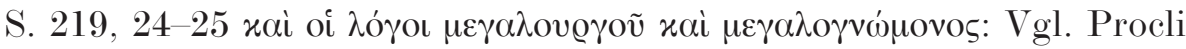

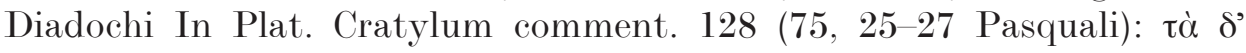

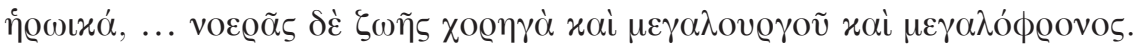




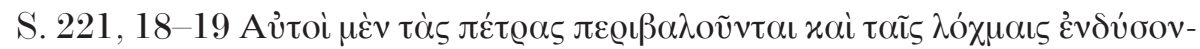

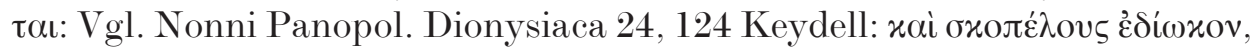

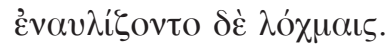

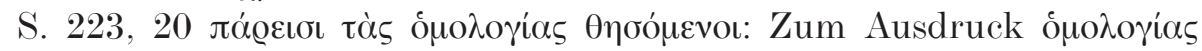

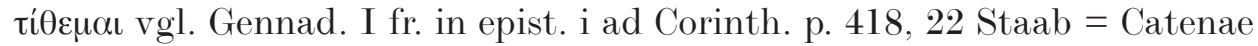
in S. Pauli ep. ad Corinth. p. 187, 24-25 (Catenae Graec. patrum in Novum

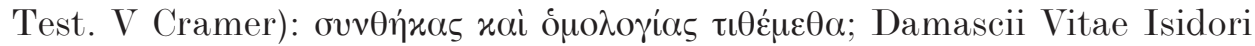
fr. 316, 2 (Epitoma Photiana 292) Zintzen = Phot. Biblioth. 352a, 12-13

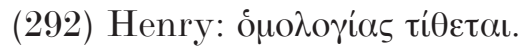

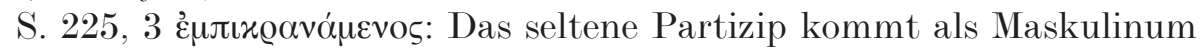
bei Philostorgius vor, Hist. eccles. VI 6a-7a (75, 21-22 Bidez-Winkelmann)

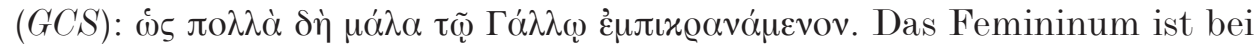
folgenden Schriftstellern belegt: Dio Cassius, Hist. Rom. 47, 8, 4 Boissevain $=$ Xiphilini epitome p. 46, 17-18 Dindorf-Stephanus $\cong$ Const. Porphyr. De virtutibus et vitiis 2, 298, 4-5 Roos $\cong$ Io. Zonar. Epit. hist. 2, 387, 9-10 Dindorf.

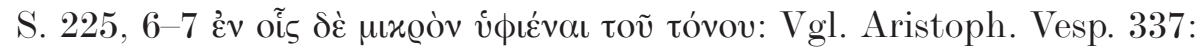

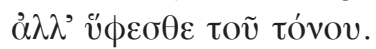

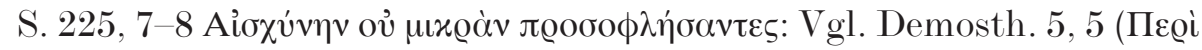

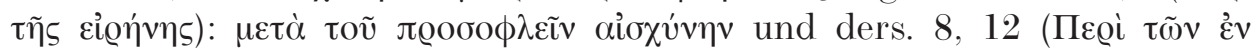

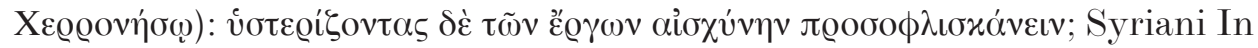

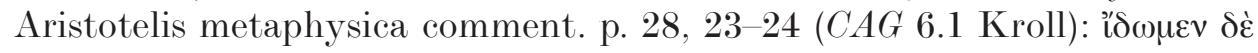

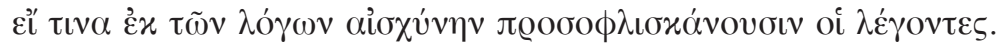

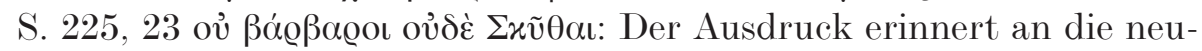

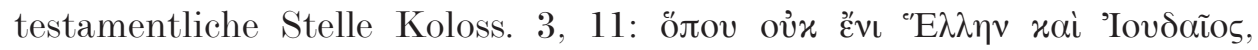

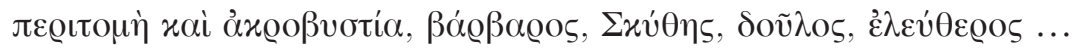

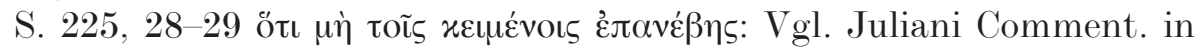

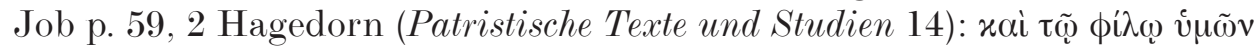

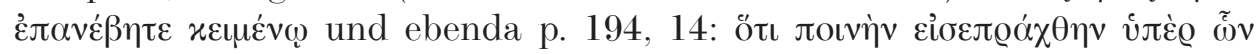

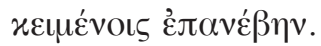

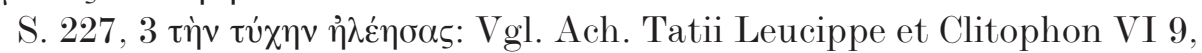

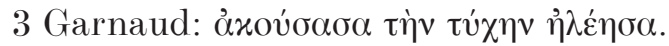

$\mu \eta \delta \dot{\varepsilon} v$ ă $\alpha \alpha v$ : Über den bekannten Spruch der sieben Weisen s. M. Tziatzi-Papagianni, Die Sprüche der sieben Weisen: Zwei byzantinische Sammlungen; Einleitung, Text, Testimonien und Kommentar (Beitr. z. Altertumskunde 51). Stuttgart 1994, 188-190 und 366-367.

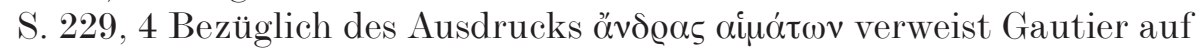

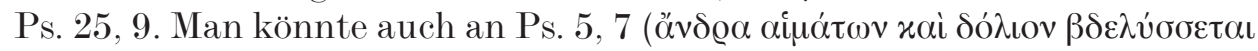
xú@ıs) denken.

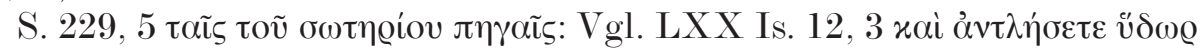

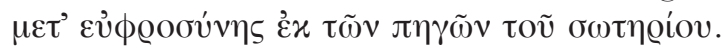


S. 229. 231 app. fontt.: Die Quelle Eph. 5, 8 bezieht sich auf Z. 5; Rom. 15, 16 auf Z. 7-8; 2 Tim. 2, 15 auf Z. 14; 3 Regn. 1, 5 auf Z. 2-3. Der Verweis auf die Affen des Archilochos wäre vollständiger, wenn man auf die ganze Epode VII verweisen würde, nämlich ,p. 64-66“".

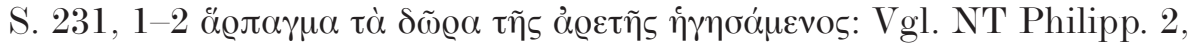

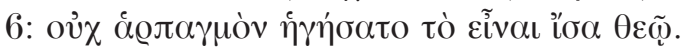

S. 231, $3{ }^{\circ} \mathrm{O} \alpha \dot{\tau} \tau о \varkappa \alpha \lambda u ́ \pi \tau \omega \nu ~ \beta \alpha \theta \dot{\varepsilon} \alpha$ : Außer Job 12, 22 (s. Gautiers app. fontt.

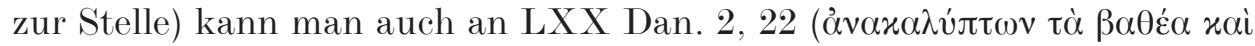

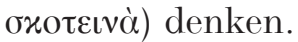

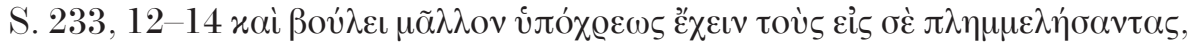

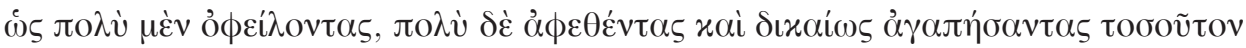

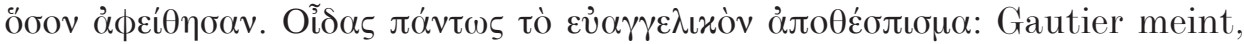
wie aus seinem apparatus fontium hervorgeht, daß es hier um die Stelle

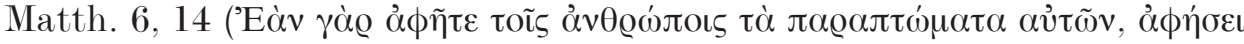

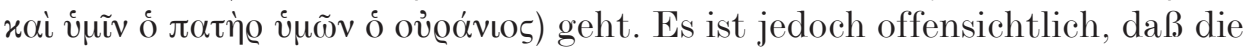
zitierte Matthäus-Stelle fast keine Beziehung zu unserem Text hat. Das

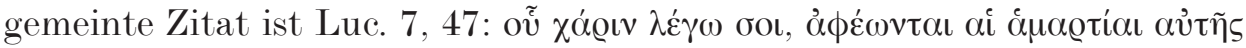

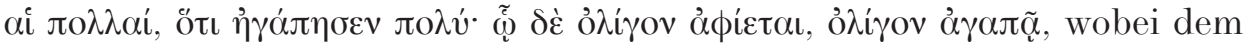
Rhetor bestimmt die ganze Perikope Luc. 7, 41-48 vorschwebte.

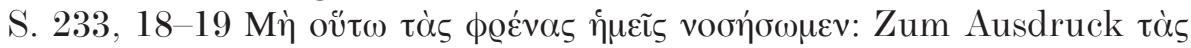
ф@évas voбeĩv vgl. Phrynichi Praeparatio sophist. (epitome) p. 91, 4 de

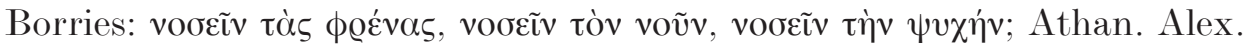

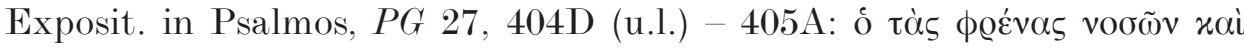

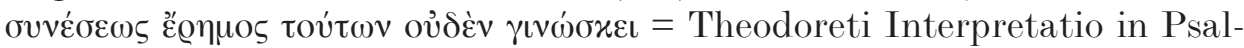
mos, PG 80, 1617C; Liban. Or. 1, 62, 3 (Libanii Opera I/1 113, 5 Foerster):

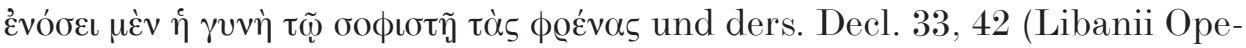

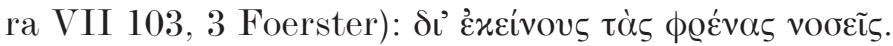

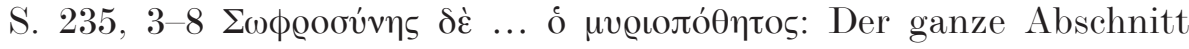

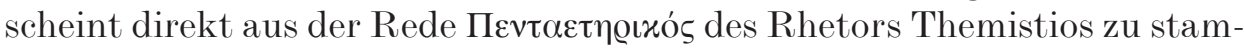
men, da die Ähnlichkeiten im Ausdruck und Sinn sehr stark und zahlreich sind $^{22}$ : Themistii Or. 8, 119d, 4 - 120a, 5 (I 179, 24 - 180, 6 Schenkl-Dow-

${ }^{22}$ Die Benutzung des Themistios durch Theophylaktos hat schon K. Prächter in seinem Aufsatz „Antike Quellen des Theophylaktos von Bulgarien“ (BZ 1 [1892] 399-414)

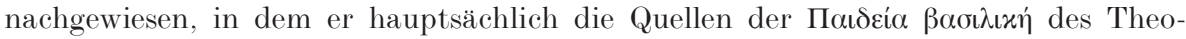
phylaktos untersucht. Den größten Teil des hier zu besprechenden Abschnitts (S. 235,

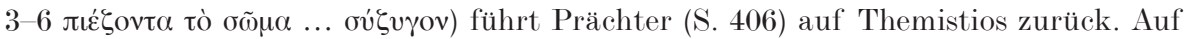
S. 403-404, 406-407 und 409 stellt er auch andere Themistios-Zitate in der Rede auf Alexios I. fest; hier erwähne ich nur diejenigen, die Prächter entgangen sind. Für weitere Quellen des Theophylaktos in derselben Rede (Xenophon, Dion Chrysostomos und Julian) s. Prächter S. 406 und 412-413. 


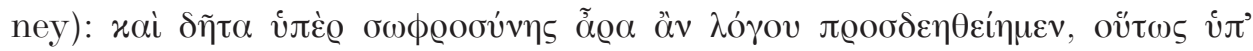

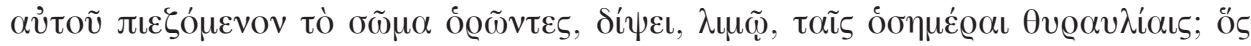

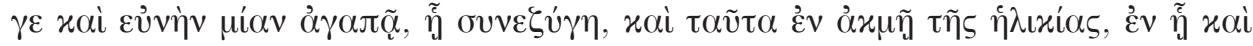

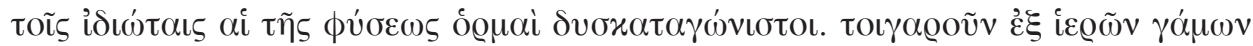

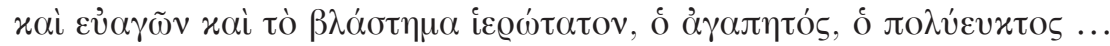

Die enge Beziehung zu Themistios ist auch unten auf S. 241, 10-12 (s. zur Stelle) offensichtlich. Ferner ist es kein Zufall, daß das sehr seltene

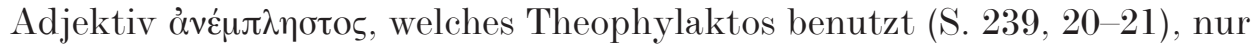
noch einmal bei Themistios als varia lectio (Or. 2, 40b), und zwar als Bestimmungswort eines ähnlichen Substantivs in dem selben Kasus (Genetiv

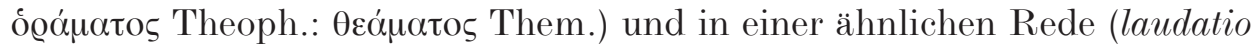
imperatoris), vorkommt.

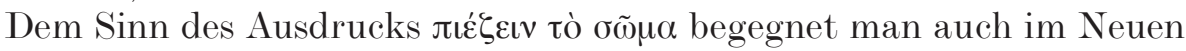

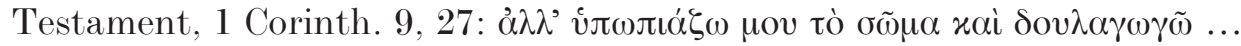

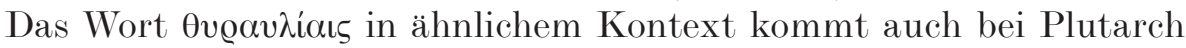
vor: Plutarchi Aetia Romana et Graeca 271B, 5-7 (II 291, 2-4 Nachstädt-

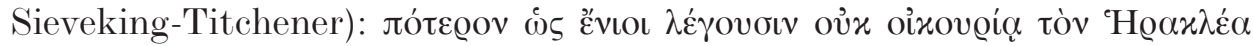

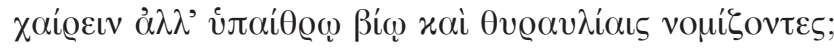

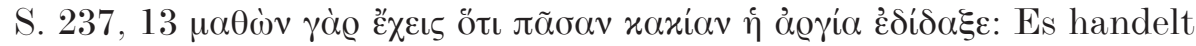
sich um ein Zitat aus der Bibel (wie aus dem einführenden Ausdruck $\mu \alpha \theta \dot{o} v$

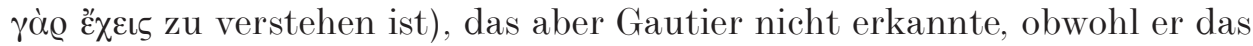

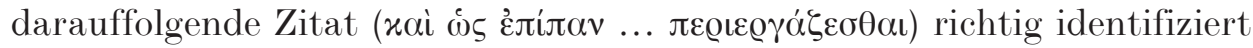

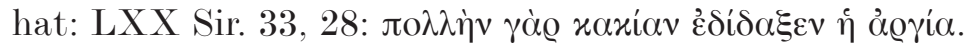

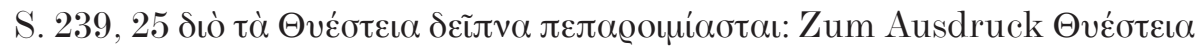

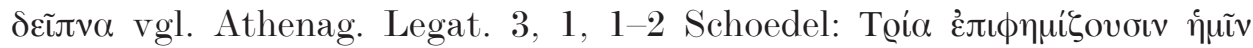

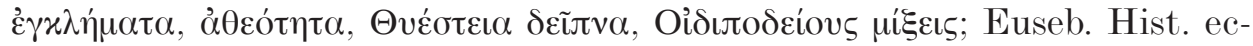
cles. 5, 1, 14 (SC 41 Bardy) = Epist. Eccles. ap. Lugdun. et Vienn. 1, 14

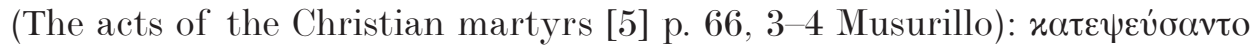

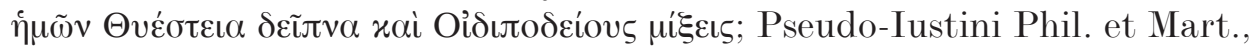
Oratio ad gentiles 3 (39 D 7) Otto (Corpus apologetarum Christ. saec. 2 III

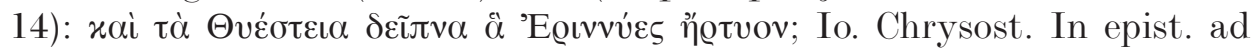

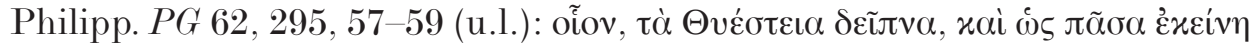

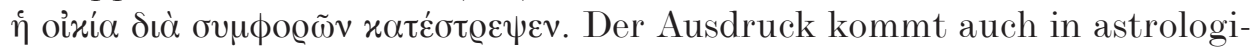
schen Werken vor, z.B. Oinop. fr. 10, 6-7 (I 394, 30s. D.-K. ${ }^{6}$ ).

S. 237. 239 app. fontt.: Hier einige kleine Versehen des Editors: Das Zitat Rom. 11, 16 bezieht sich auf die Zeilen 21-22 sowie 24-25 (S. 237) des Theophylaktos-Textes und Prov. 31, 29 auf Z. 15-16 (S. 239).

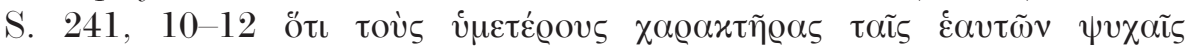

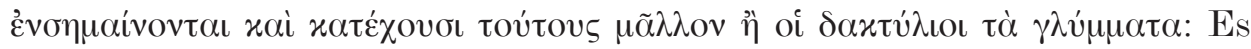
handelt sich um ein fast wortwörtliches Zitat aus Themistios, aus dem Theophylaktos auch oben (s. zur S. 235, 3-8) einen längeren Abschnitt 


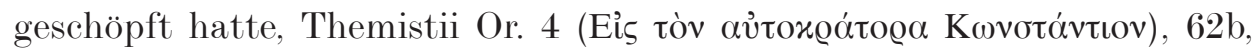

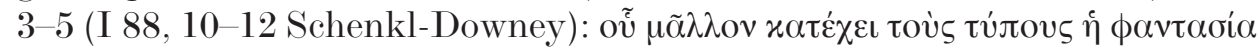

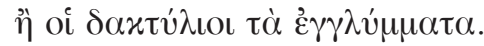

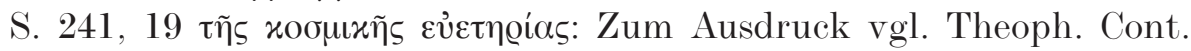

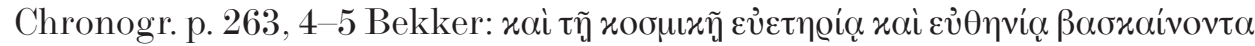

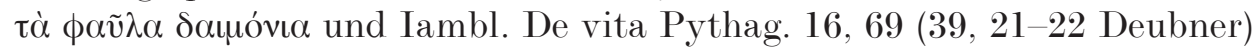

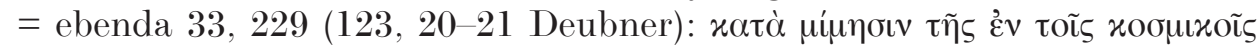

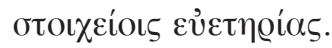

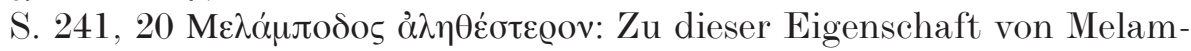
pus vgl. Scholia in Hes. opera et dies 800 bis, 5-8 (Poetae minores Graeci II

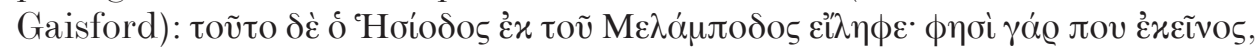

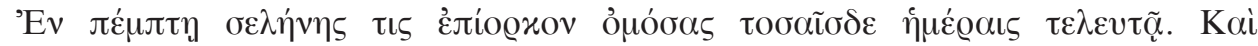

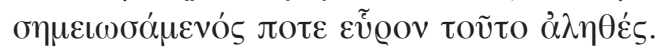

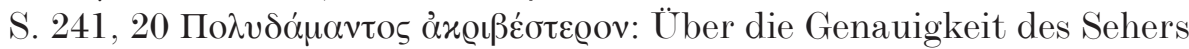
Polydamas konnte ich nichts Ausdrückliches finden. Aus folgender Eustathios-Stelle geht hervor, daß Polydamas normalerweise als sehr guter und genauer Seher galt: Eust. Comment. ad Homeri Il. 900, 57s. (3, 379, 19-21

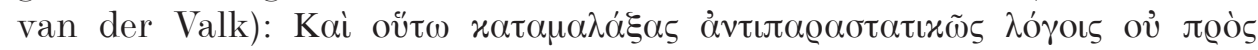

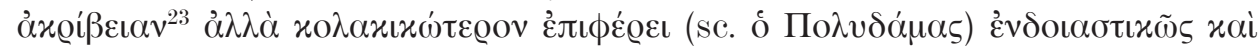

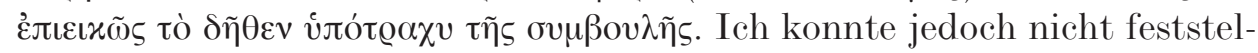
len, ob Theophylaktos und Eustathios auf einer gemeinsamen Quelle beruhen.

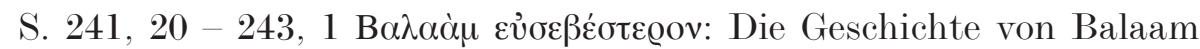
steht im Alten Testament (Numeri 22-24 et 31, 8. 16). Balaam war ein Weissager, der nach der Vision eines Engels für kurze Zeit fromme Prophezeihungen ausgesprochen hat. Danach zeigte er sich aber wieder gottlos und respektlos und wurde deshalb von den Israeliten getötet. Seine Gottund Respektlosigkeit wird in folgenden Texten betont: Philonis De migratione Abrahami 113 (Philonis Alex. opera II 290, 10-13 Wendland); Constitutiones Apostol. 8, 2, 2. 5 (SC 336 Metzger); Theodoreti De quaestion. ambiguis in libr. Regn. et Paralip. $P G$ 80, 589D (u.l.) - 592A Mich. Glycae Annales II 330, 12-14 Bekker.

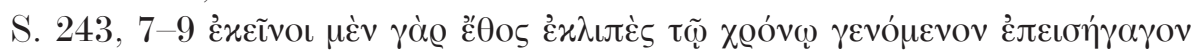

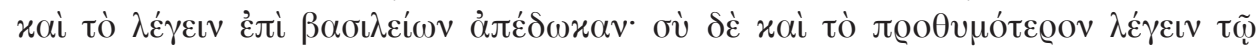

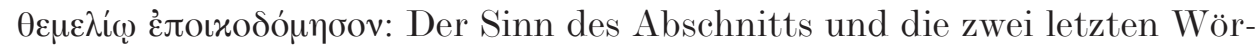

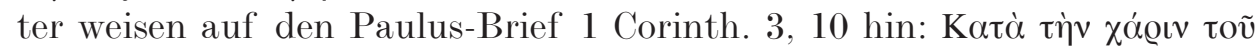

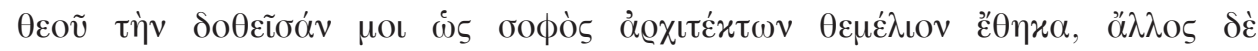

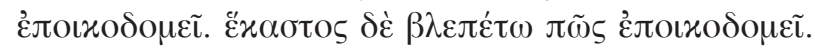

${ }^{23}$ Dies ist so zu verstehen, daß Polydamas in diesem Fall ausnahmsweise nicht genau, sondern eher schmeichelnd gesprochen hat. 
Zur panegyrischen Rede des Theophylaktos von Achrida auf Kaiser Alexios I. 203

\section{$\mathrm{ANHANG}^{24}$}

Nach der systematischen Kollation der Ausgabe Finettis hat es sich als lohnend erwiesen, folgende Stellen der Rede zusätzlich bzw. ergänzend zu diskutieren ${ }^{25}$. Es ist anzunehmen, daß Finetti (an den Stellen, an denen er nichts notiert) den Text des Monac. gr. 66, von möglichen Lesefehlern abgesehen, treu wiedergibt. Deshalb ist hier im folgenden, wenn es sich nicht ausdrücklich um eine Korrektur Finettis handelt, die Rede von Lesarten des Monacensis.

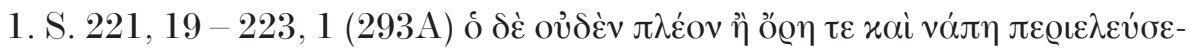

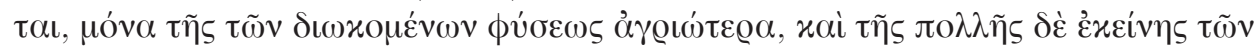

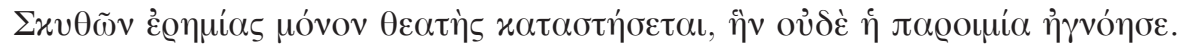

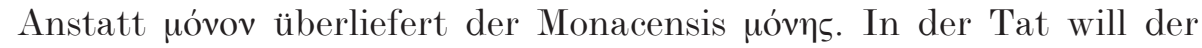
Redner nicht etwa sagen, daß man (ó $\delta \dot{\varepsilon})$ nur Zuschauer werden wird ( $\mu$ óvov

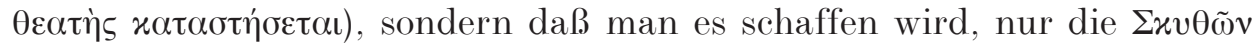

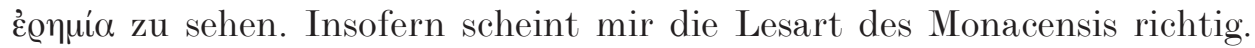
(Vgl. auch den Gebrauch von $\mu$ óva kurz davor im selben Abschnitt: ọ̋ $\eta \varepsilon$

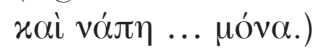

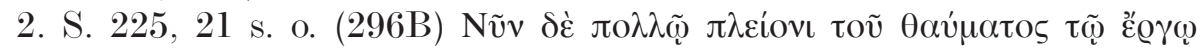

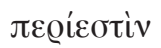

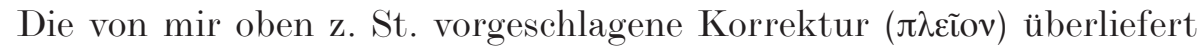
auch der Monacensis.

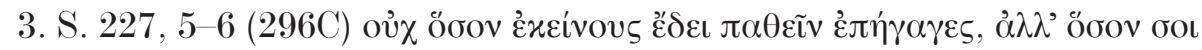

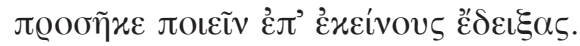

Die Stelle des Pronomens éxદívous (im ersten Teil des Vergleichs) zeigt, daß das entsprechende Pronomen ool (im zweiten Teil) eigentlich betont, also orthotoniert werden sollte. Im Monacensis steht richtig ooi.

${ }^{24}$ Nachdem ich die vorstehenden Ausführungen abgeschlossen hatte, warf ich aus Neugier nur einen Blick in Finettis Ausgabe (s. o. A. 2). Dabei stellte ich fest, daß eine systematische Kollation dieser Ausgabe nicht uninteressant wäre. Die Ergebnisse dieser Kollation präsentiere ich im vorliegenden Anhang. Darüber hinaus ist zu bemerken, daß Finetti an einigen Stellen besser interpungiert hat, z.B. (mit Hochpunkt): S. 237, 19

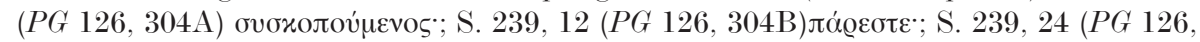

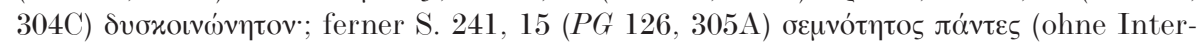
punktion zwischen den beiden Wörtern). Außerdem ist es ihm gelungen, die Quellen von zwei Stellen zu identifizieren, die in Gautiers beiden Ausgaben fehlen: S. 227, 10 (PG 126, 296D) LXX Ezech. 33, 11 und S. 233, 14 (PG 126, 300C) NT Luc. 7, 43 (hier allerdings unvollständig und ohne den wichtigsten Teil des Zitats zu erkennen, s. o. S. 200 zur S. $233,12-14$ ).

${ }^{25}$ Verweis und Text gebe ich nach Gautiers Ausgabe; es folgt jeweils in Klammern der Verweis auf Finetti (die Kolumnenzahl in $P G$ 126). 


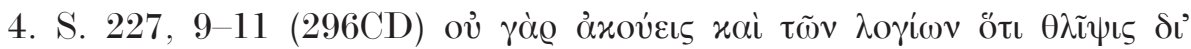

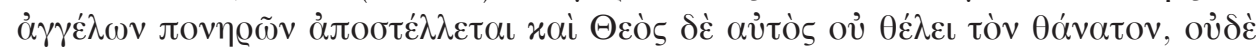

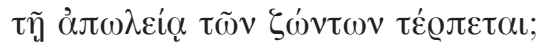

In dieser Textpartie zitiert der Redner aus drei verschiedenen Quellen:

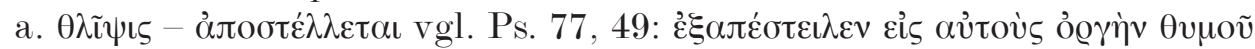
$\alpha$

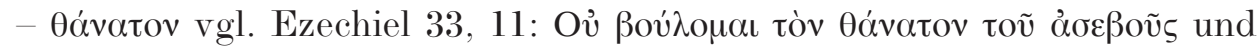

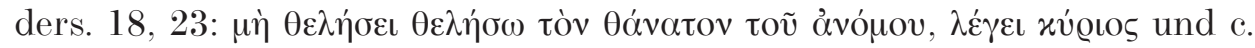

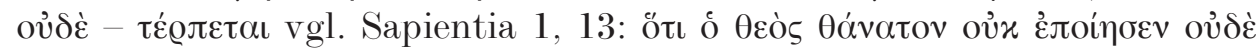

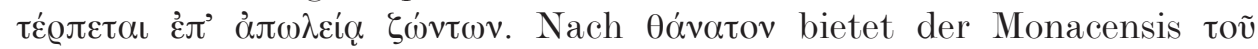

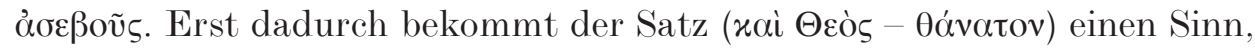
bzw. wird das Zitat (b) vervollständigt. Daß der Schreiber die Wörter von

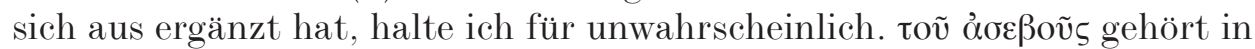
den Text. Gautier erwähnt als Quelle hierzu eine andere Stelle aus Ezechi-

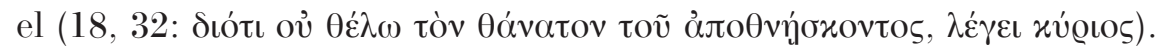

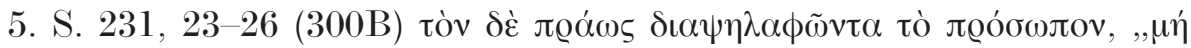

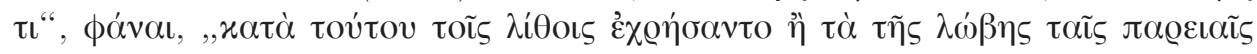

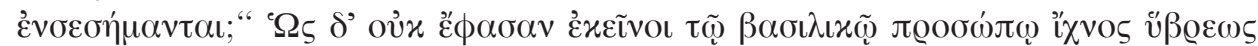

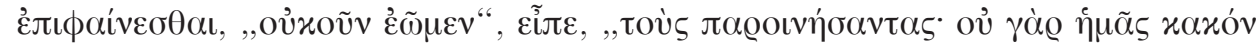

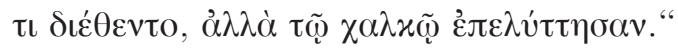

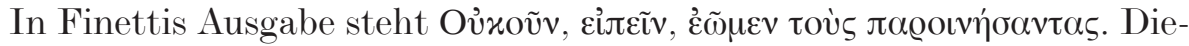
sen Text überliefern sowohl der Monacensis als auch L (wie aus Gautiers kritischem Apparat z. St. ersichtlich ist). Wenn man den ganzen Abschnitt aufmerksam liest, merkt man, daß die Worte des Konstantinos einige Zeilen davor durch den Infinitiv dóvol eingeführt sind. Auch hier ist also eine ähnliche Syntax zu erwarten. Der überlieferte (und dem vorangehenden

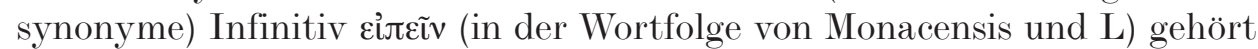

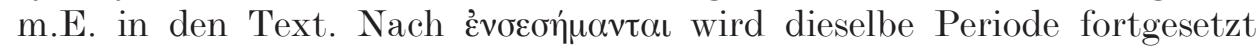
(... $\omega \varsigma)$.

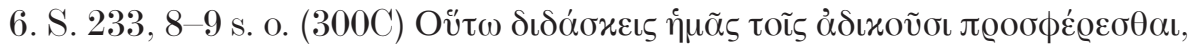

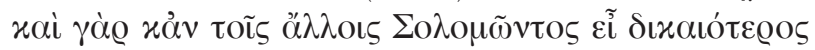

In Finettis Ausgabe steht (wie in der früheren Ausgabe von Gautier) richtig xỏv. Außerdem hat Finetti mit Recht (wie Gautier in seiner ersten Ausgabe) nach $\pi \varrho o \sigma \phi \varepsilon ́ \varrho \varepsilon \sigma \theta \alpha$ einen Punkt gesetzt.

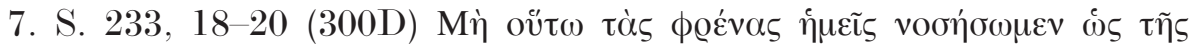

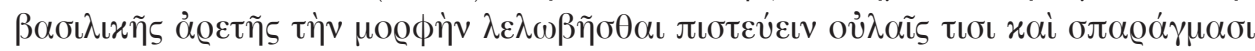

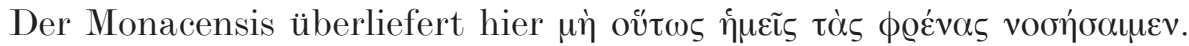
Diese Wortfolge, die laut Gautiers kritischem Apparat auch in L belegt ist, gehört m.E. in den Text. Über den Modus läßt sich jedoch nicht einfach entscheiden. Sowohl der adhortative Konjunktiv als auch der kupitive Optativ sind möglich. Ersterer ist vielleicht vorzuziehen, da er als Modus 
des Wollens gebräuchlicher ist: Im elektronischen TLG sind 4 (weitere)

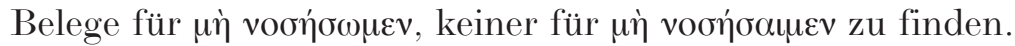

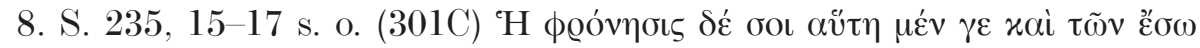

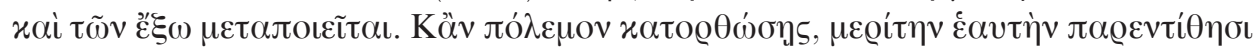

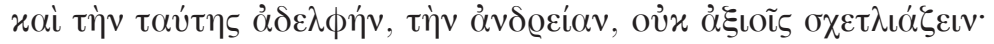

Einige der oben z. St. erwähnten Vorschläge weist auch der Monacensis

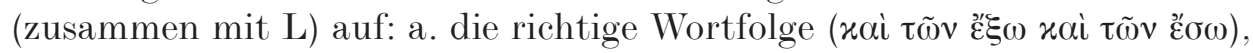

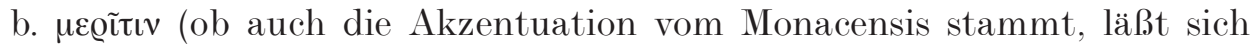
nicht mit Sicherheit sagen, sehr wahrscheinlich jedoch überliefert der Co-

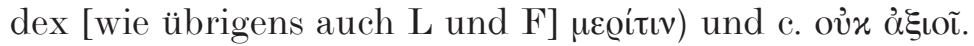

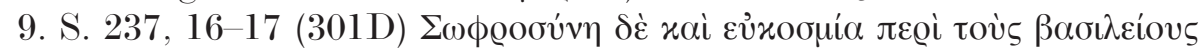

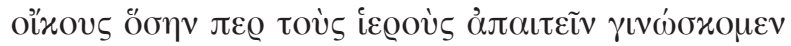

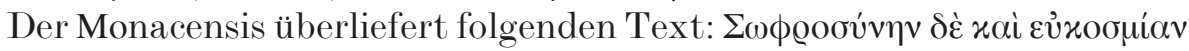

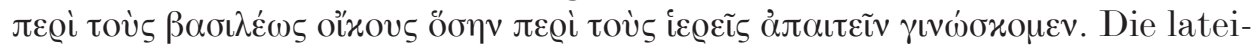
nische Übersetzung hierzu (in $P G$ ) lautet: Moderationem vero et decentiam tantam circa regias ades, quantam circa sacerdotes te exigere novimus. Zunächst scheinen beide Lesarten, $\beta \alpha \sigma i \lambda \varepsilon \dot{\omega} \omega \varsigma$ und $\beta \alpha \sigma i \lambda \varepsilon i ́ o v \varsigma$, richtig zu sein. Man könnte allerdings behaupten, daß $\beta \alpha \sigma \iota \lambda \varepsilon \dot{\varepsilon} \omega \varsigma$ die lectio difficilior ist,

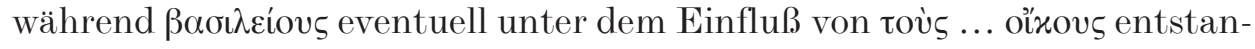

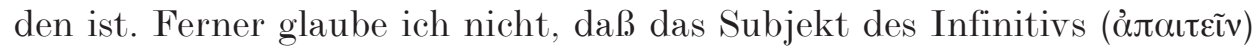
oغ̀ sein kann (so nach der Übersetzung), weil a. oغ̀ nicht automatisch zu ergänzen ist und b. in der Fortsetzung der Rede gesagt wird, daß das Vor-

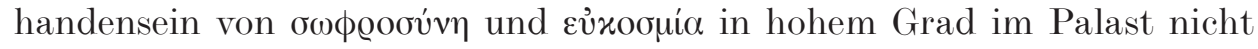

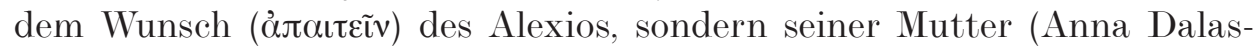

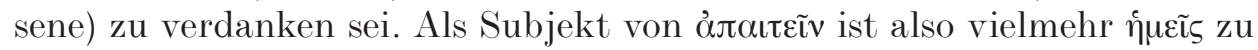

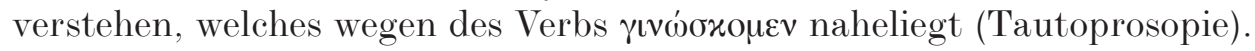

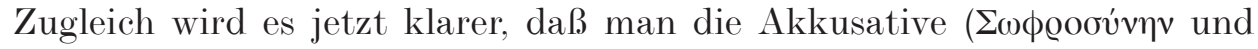

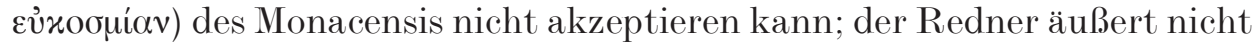

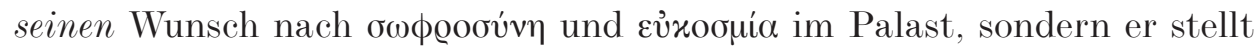

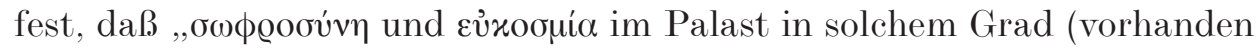
sind), wie wir sie von ... zu verlangen wissen“. Im griechischen Text

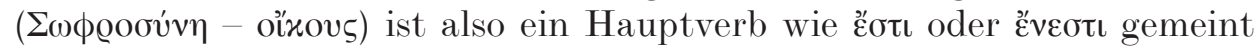

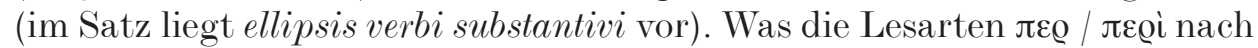

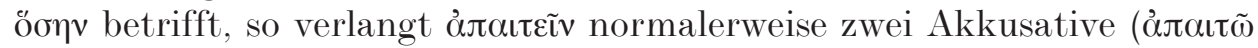

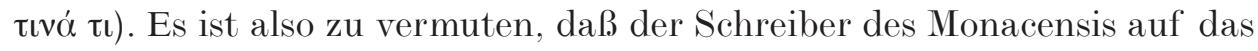
zweite $\pi \varepsilon \varrho i$ unter dem Einfluß des ersten gekommen ist. Das letzte hier zu besprechende Problem ist das zweite Glied des Vergleichs. Sind es die Kirchen (ígoùs [gemeint ist oíxovs]), wie es Gautier (nach F) will, oder die

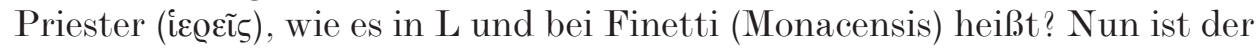
Redner ein Erzbischof, der über Priester verfügt; insofern wäre es verständ-

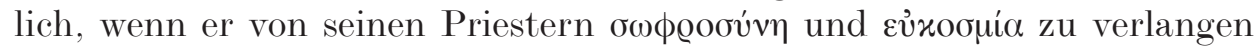




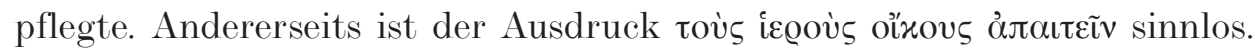
Demnach ist die Lesart iع@eĩs vorzuziehen. Erst dadurch wird auch das

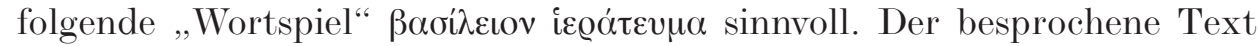
lautet nun wie folgt:

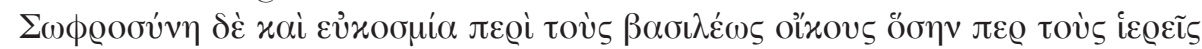

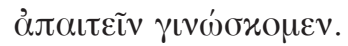

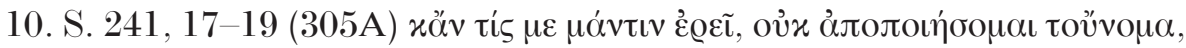

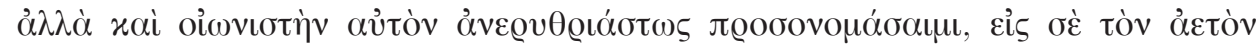

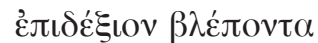

Der Monacensis überliefert die von mir vorgeschlagene Korrektur avitòv.

Nachdem oben festgestellt wurde, daß der Monacensis mindestens an

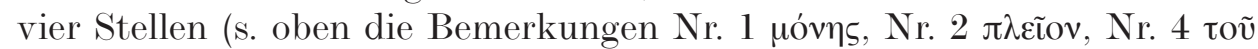

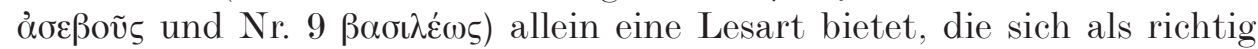
erwiesen hat, während L und F eine falsche Lesart (sei es die gleiche oder jeweils eine andere) überliefern, entsteht jetzt die Frage, ob die Behauptung von Gautier (S. 43), daß der Monacensis ein Apographon von L sei, aufrecht erhalten werden darf. Gautier begründet seine Annahme nicht, auf der nächsten Seite sagt er sogar (in Bezug auf ein anderes Werk von Theophylaktos), der Monacensis sei ein Apographon von F. Wenn wir ausschließen, daß der Schreiber des Monacensis auf die erwähnten richtigen Lesarten zufällig oder durch eigene Korrektur gekommen sein kann, kommen wir zur Schlußfolgerung:

Der Monacensis ist kein Apographon von L (daß die beiden an vielen Stellen denselben Fehler aufweisen, beweist nur, daß die beiden Handschriften enger miteinander verwandt sind als eine der beiden mit F), somit hat er den Wert eines unabhängigen Überlieferungsträgers und muß folglich als solcher in künftigen Editionen mit herangezogen werden. 